\title{
Laparoscopia en la fibrosis retroperitoneal
}

\author{
L.A. Fariña Pérez, John dos Santos, J. Cambronero Santos, E.R. Zungri Telo \\ Servicio de Urología. Centro Médico POVISA. Vigo (Pontevedra).
}

Actas Urol Esp 2005; 29 (4): 434-435

$\mathrm{P}_{\mathrm{t}}^{\mathrm{sec}}$ ese a que se sigue recomendando en los textos, la biopsia de la fibrosis retroperitoneal (FRP) para excluir un origen maligno, no es una costumbre habitual, puesto que muchas veces puede suponerse por la historia clínica y los medios de imagen el carácter idiopático o benigno, que supone la mayoría de los casos, $y$, por otra parte, el ensayo terapéutico con corticoides tiene baja morbilidad en la fibrosis de causa maligna. Sin embargo, la biopsia debería indicarse cuando se sospecha malignidad, es decir, en pacientes con antecedente de neoplasia de posible diseminación retroperitoneal, y cuando la fibrosis incluya las áreas retroaórticas, -en cuyo caso ha de considerarse el diagnostico de linfoma-, o se sitúe en lugares no típicos del trayecto ureteral $^{1}$. Las indicaciones de ureterolisis en caso de resistencia a los corticoides o de compromiso ureteral grave son más generalmente asumidas.

En algunos casos puntuales informados en la bibliografia, la biopsia retroperitoneal y la ureterolisis mediante laparoscopia pudieron sustituir a las técnicas de cirugía convencional para el diagnostico y el tratamiento de la FRP, con menor morbilidad, menor coste hospitalario y de convalecencia $^{2-5}$.

Como tuvimos ocasión de comprobar en una mujer de 62 años diagnosticada de FRP benigna, secundaria a periaortitis, con compromiso del uréter izquierdo, la biopsia y desplazamiento del uréter de la fibrosis que lo atrapa es posible mediante laparos- copia. Con la paciente en decúbito lateral y tres trócares situados en región paraumbilical (óptica), subcostal e iliaca, el colon fue decolado y se identificó, en la porción más distal de la masa de fibrosis, el uréter con un catéter doble $\mathrm{J}$ en su interior. Trabajando con el endodisector en ángulo recto y la endotijera, se abrió la carcasa de fibrosis que rodeaba el uréter, quedando éste libre con cierta facilidad en una longitud de unos 10-12 cm, momento en el que el catéter doble $\mathrm{J}$ resultó muy útil, al dar consistencia a un uréter muy adelgazado, que pudo entonces ser manipulado con menos riesgo. El tejido fibrótico se biopsió en varios puntos con la endotijera, encontrando en el estudio intraoperatorio tejido inflamatorio benigno. A continuación el uréter fue desplazado lateralmente, fijándolo sobre el psoas, en una posición alejada de la fibrosis. Esta paciente estuvo hospitalizada 36 horas y está ahora bajo tratamiento con corticoides (Fig 1).
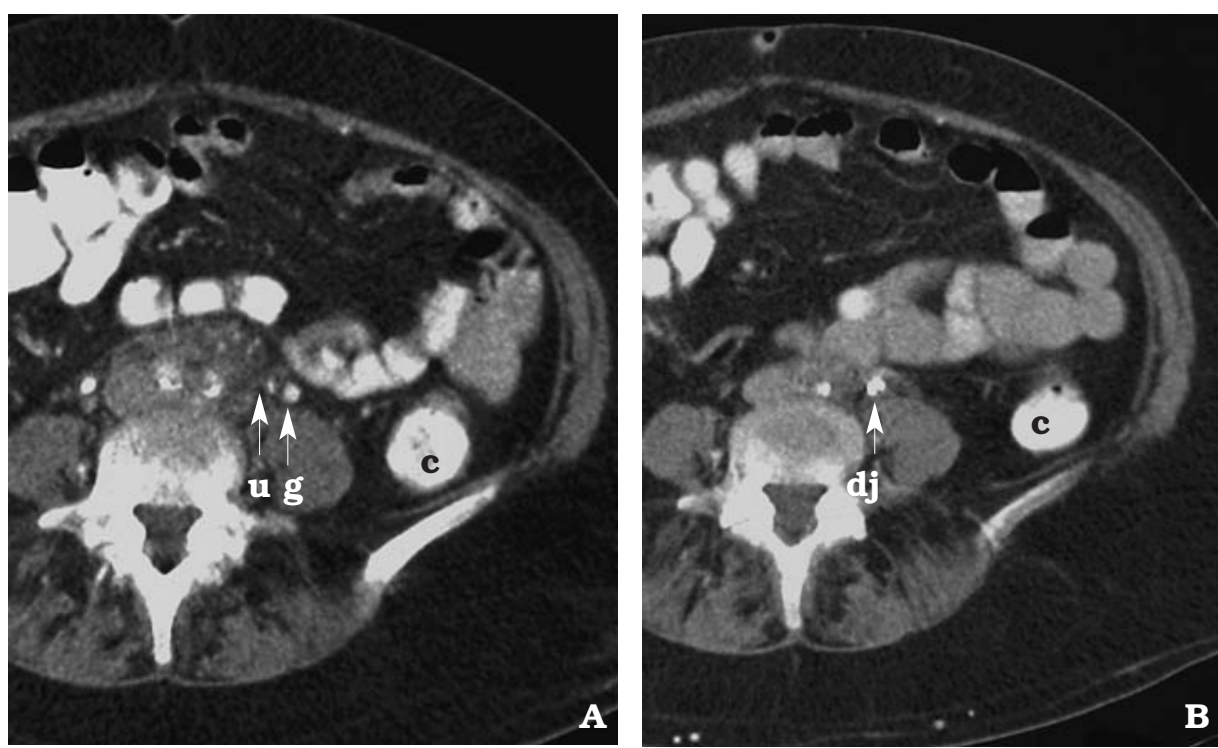

Figura 1. Tomografía computarizada antes (A) y después (B) de ureterolisis laparoscópica y tratamiento corticoideo. Obsérvese en $B$ el uréter (con doble $J$ en su interior) desplazado de la fibrosis periaórtica residual $(u=$ uréter, $g=$ vena gonadal, $d j=$ catéter doble $J, c=$ colon). 
Revisando la bibliografía escrita sobre esta técnica, es por ahora imposible sacar conclusiones generales sobre cuándo abordar mediante laparoscopia una enfermedad de causa tan diversa y con formas tan variables de afectación ureteral. Parece que, por el momento, debe considerarse un procedimiento de laparoscopia avanzada, por las dificultades de identificación de las estructuras afectadas, por el riesgo de desvascularizar el uréter, que queda con una pared muy fina y a merced de la irrigación proveniente de sus extremos proximal y distal. Por último, en caso de que los hallazgos preoperatorios lo hicieran necesario, por las dificultades que entraña realizar una disección bilateral, una omentoplastia o una intraperitonización del uréter.

\section{REFERENCIAS}

1. Kava BR, Russo P, Conlon KC. Laparoscopic diagnosis of malignant retroperitoneal fibrosis. J Endourol 1996; 10:535-538.

2. Matsuda T, Arai Y, Muguruma K, Uchida J, Shichiri Y, Komatz Y. Laparoscopic ureterolysis for idiopathic retroperitoneal fibrosis. Eur Urol 1994;26:286-290.

3. Elashry OM, Nakada SY, Wolf JS Jr, Figenshau RS, McDougall EM, Clayman RV. Ureterolysis for extrinsic ureteral obstruction: a comparison of laparoscopic and open surgical techniques. J Urol 1996;156:1403-1410.

4. Demirci D, Gulmez I, Ekmekcioglu O, Sozuer EM, Keklik E. Intraperitonealization of the ureter during laparoscopic ureterolysis: a modification of the technique. J Urol 2001;165:180-181.

5. Fugita OE, Jarrett TW, Kavoussi P, Kavoussi LR. Laparoscopic treatment of retroperitoneal fibrosis. J Endourol 2002;16:571-574.

Dr. L.A. Fariña Pérez

Centro Médico Povisa

Salamanca, 5 - 36211 Vigo 